\title{
„Wölfe“ im Palmengarten
}

\author{
Hilke Steinecke
}

\begin{abstract}
The Palmengarten hosts many different insect species. Many of them are predators or parasites of other insects. They are fine examples of the fact that, even in a garden, there are diverse relationships between different species and biodiversity should be promoted. Two different insect species can be observed in the Palmengarten, both of which are named Bienenwolf (bee wolf). These include the beetle Trichodes apiarius and the solitary wesp Philanthus triangulum. Both live in the Palmengarten, preferably in the steppe and in the rock garden. During the summer they are regularly found on Miss Willmott's ghost (Eryngium giganteum) and other Eryngium species.
\end{abstract}

\section{Zusammenfassung}

Im Palmengarten sind viele verschiedene Insektenarten zu beobachten. Viele von ihnen leben als Räuber oder Parasiten von anderen Insekten. Sie sind ein schönes Beispiel dafür, dass in einem Garten vielfältige Beziehungen zwischen verschiedenen Arten bestehen und die Artenvielfalt gefördert werden sollte. Nicht selten sind im Palmengarten zwei ganz unterschiedliche Insekten zu beobachten, die beide den deutschen Volksnamen „Bienenwolf“ tragen. Es handelt sich um den Käfer Trichodes apiarius und die Grabwespe Philanthus triangulum. Beide halten sich im Palmengarten bevorzugt in der Steppenanlage sowie im Steingarten auf. Regelmäßig sind sie im Sommer auf Mannstreu (Eryngium giganteum u. a.) anzutreffen.

\section{Einführung}

Mit räuberisch lebenden Tieren verbinden viele zunächst große Säugetiere wie Wölfe, Löwen oder Tiger. Einige kleinere wirbellose Tiere, die ebenfalls

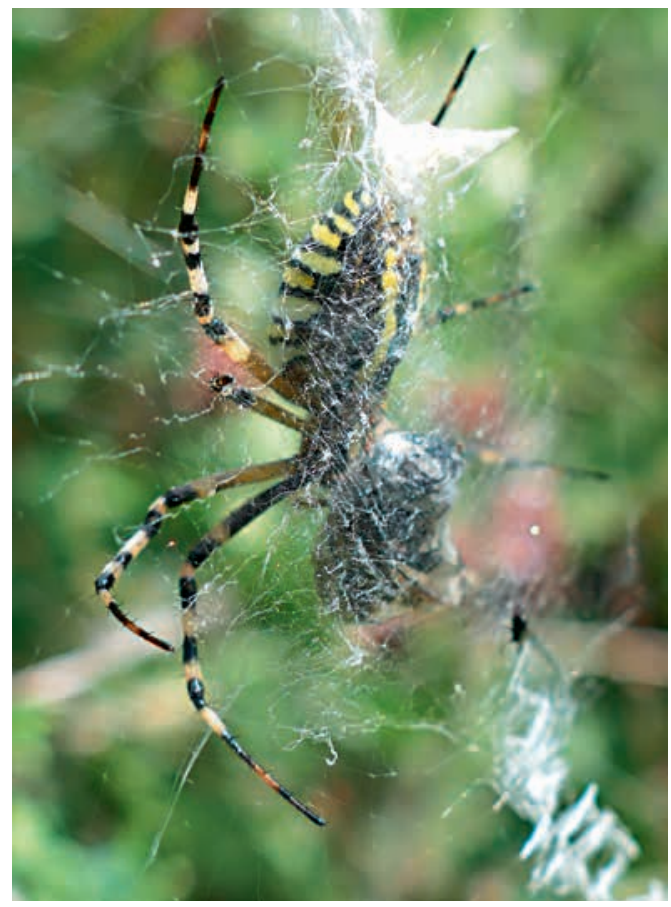

Abb. 1: „Kleines Raubtier“ mit gefährlichem Namen in der Steppenwiese: Zebra- oder Tigerspinne (Argiope bruennichi). (Foto: H. Steinecke) räuberisch leben, erinnern mit ihren deutschen Bezeichnungen an diese Tiere. Vor allem wegen ihres gelb-schwarzen Streifenmusters und vielleicht zusätzlich wegen ihrer räuberischen Lebensweise wird Argiope bruennichi, die selten auch im Palmengarten anzutreffen ist, als Tigerspinne (auch Wespen- oder Zebraspinne) bezeichnet. Wolfsspinnen (Lycosidae) leben je nach Art in Erdhöhlen, die sie mit Spinnseide auskleiden, oder zwischen Steinen in der Krautschicht. Dort legen sie ihre Gespinste an und warten auf Beute. Ameisenlöwen sind die Larven der libellenähnlichen Ameisenjungfern (Myrmeleontidae). Die Larven bauen im lockeren und trockenen Substrat Fangtrichter, an dessen Grund sie sich verstecken. Sie fangen Ameisen und andere Gliedertiere, die in die Trichter geraten. Zwei markante „Wölfe“ unter den Insekten sind eine Wespen- sowie eine Käferart. Beide tragen die deutsche Bezeichnung Bienenwolf und sind im Palmengarten häufig zu beobachten. In den Sommermonaten kann man sie vor allem in der artenreichen Steppenanlage sowie im Steingarten an verschiedensten Futterpflanzen finden.

\section{Philanthus triangulum}

Der Bienenwolf (Philanthus triangulum) ist eine auffällige und relativ große solitär lebende Grabwespe (Körperlänge des Weibchens bis $18 \mathrm{~mm}$, die Männchen sind etwas kleiner). Der Körper ist 


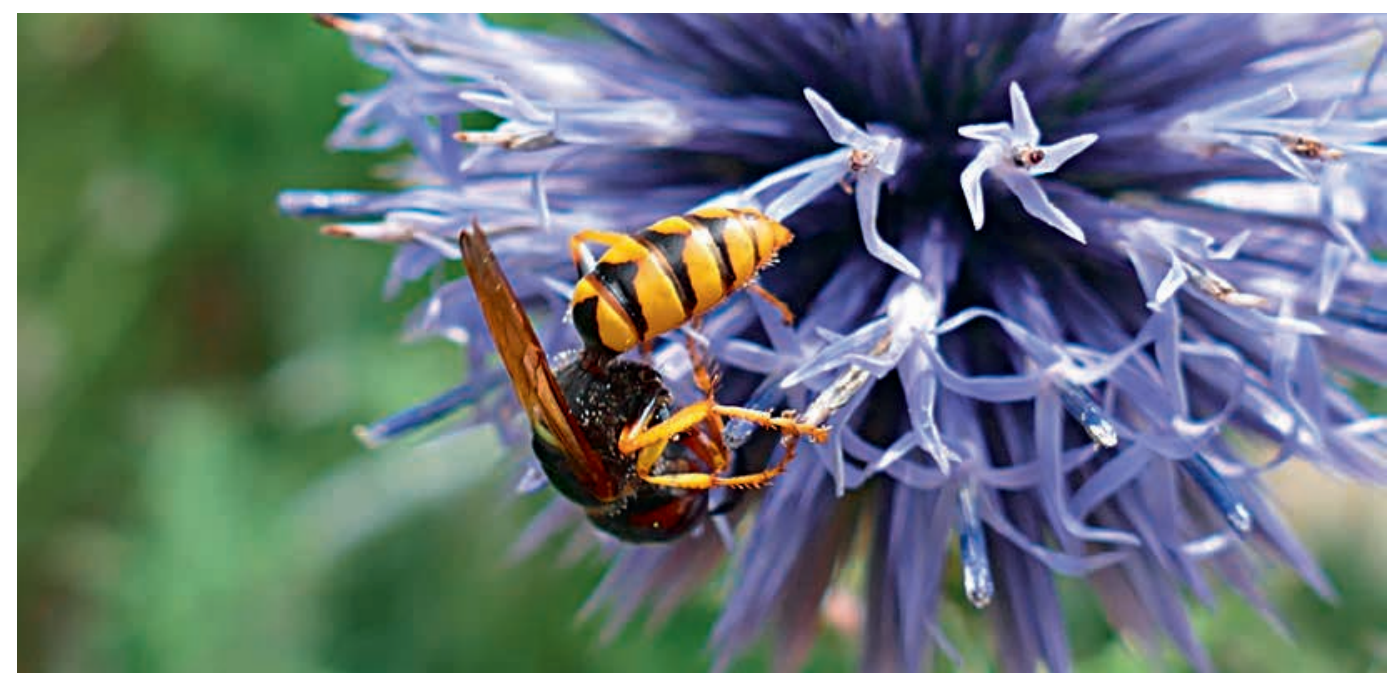

Abb. 2: Kugeldisteln (Echinops) sind bei Philanthus triangulum sehr beliebt (Foto: H. STEinecke)

schwarz-gelb gemustert, weshalb man sie auf den ersten Blick auch für eine kräftige Echte Wespe halten könnte. Die schwarzgefärbten Bereiche auf dem Hinterleib haben eine dreieckige Form, deshalb der Artbeiname triangulum. Auffällig ist bei den Männchen die helle Gesichtsmaske. Der Bienenwolf ist in weiten Bereichen Europas bis nach Sibirien verbreitet.

Die ausgewachsenen Insekten besuchen diverse Blüten und trinken Nektar. Die Larven dieser Grabwespe dagegen ernähren sich von Honigbienen, die von den Weibchen gefangen und dann in das Nest gebracht werden. Die weiblichen Bienenwölfe lauern ihrer Beute meist dann auf, wenn diese auf den Blüten mit dem Nektar-oder Pollensammeln beschäftigt ist. Die gefangene Biene wird durch einen Stich betäubt, so dass sie sich nicht weiter wehren kann. Der Bienenwolf drückt auf den Magen der Biene, woraufhin sie Nektar abgibt, der dann vom Bienenwolf gefressen wird. Die Biene wird anschließend gedreht und auf dem Rücken in die Brutröhre geschoben. Die Beute wird vom Bienenwolf-Weibchen mit einer Mischung aus neun verschiedenen Antibiotika eingerieben, damit sie frisch bleibt, bis sie schließlich von den Larven gefressen wird. Die Antibiotika stammen von Streptomyces-Bakterien, die symbiontisch in den hohlen Antennen der Wespe leben.
Früher war der Bienenwolf ziemlich häufig und bei Imkern nicht gerade beliebt. Stellenweise wurde er sogar bekämpft. Mittlerweile sind seine Bestände aber stark geschrumpft, da seine bevorzugten Lebensräume wie Steppen, Heiden und Magerwiesen rückläufig sind. Umso erfreulicher ist es, dieses auffällige Insekt den ganzen Sommer über regelmäßig im Palmengarten beobachten zu können. Besonders angezogen werden sie im Hochsommer von verschiedenen Mannstreu-Arten wie Eryngium giganteum und E. planum. Im Spätsommer sieht man sie häufig auf Solidago-Arten. Ende September sterben die Imagines, die Larven überwintern und spinnen sich einen Kokon. Auch der Kokon ist mit Streptomyces-Bakterien infiziert. Nach der Puppenruhe schlüpfen etwa ab Juni des Folgejahres die neuen Bienenwölfe. Beim Verlassen des Kokons nimmt das Weibchen die Streptomyces-Bakterien in ihre Antennen auf, um dafür gerüstet zu sein, die erlegte Beute frisch zu halten.

\section{Trichodes apiarius}

Dieser farbenfrohe Bienenwolf ist ein Käfer aus der Familie der Buntkäfer (Cleridae). Er wird auch Gemeiner Bienenkäfer, Immenwolf oder Immenkäfer genannt. Diese Art hat ein weites Verbreitungsgebiet von Nord-Afrika über Europa bis nach Kleinasien. Stellenweise ist dieser Käfer selten geworden, in Deutschland vor allem im Norden und Osten. 


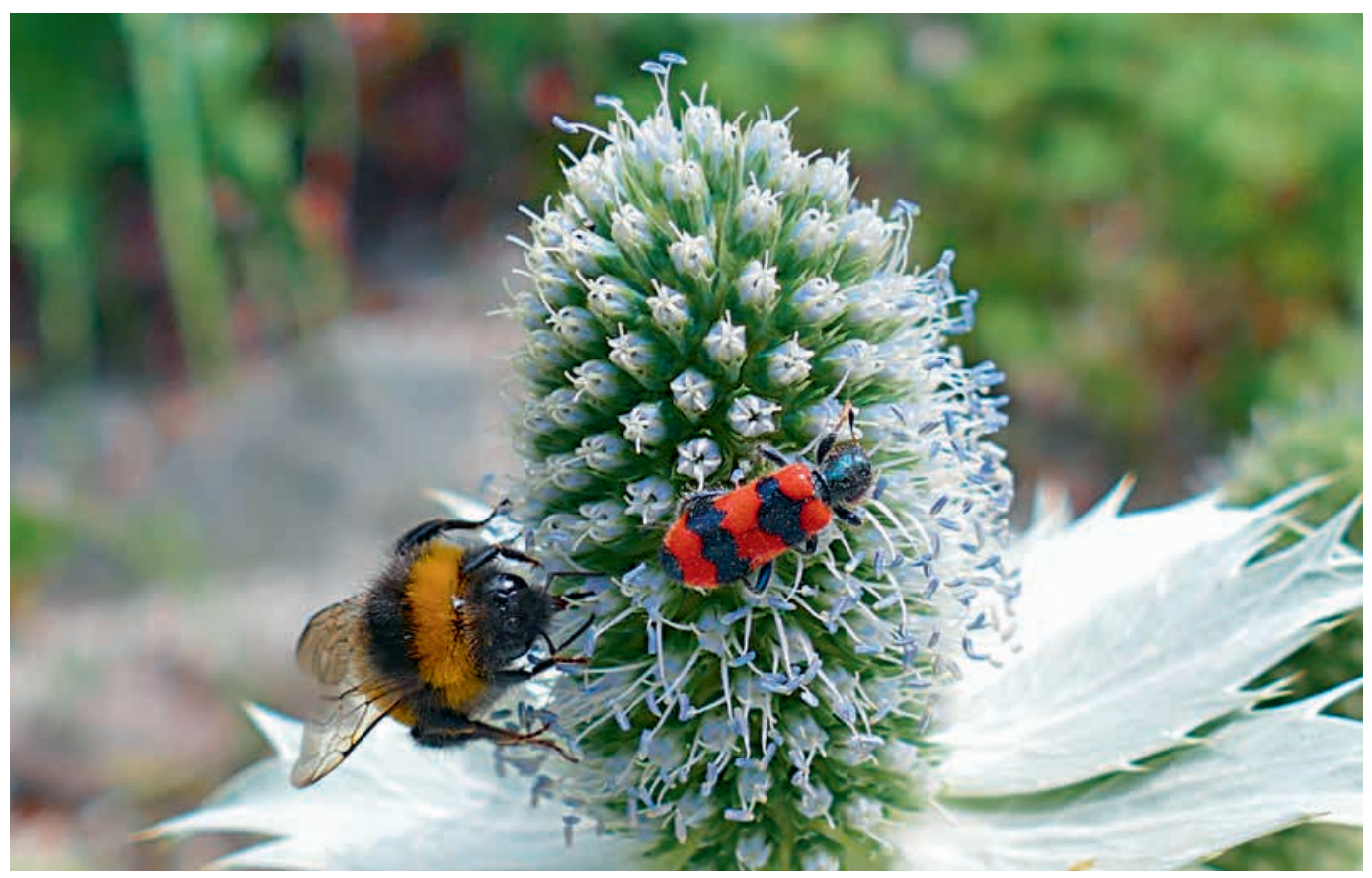

Abb. 3: Dieser Bienenwolf (Trichodes apiarius) ist ein auffällig schwarz-rot gefärbter Käfer. Auch er ist wie Hummeln häufig an Eryngium planum anzutreffen. (Foto: H. STEINeCKe)

Er ist wärmeliebend und hält sich bevorzugt auf Trockenrasen oder in sonnigen Gärten auf.

Der Käfer wird 9-16 mm lang, ist auf dem Körper behaart und zeigt eine auffällige schwarz-rote Bänderung. Etwa von Mai bis Juli sind die Käfer unterwegs und vor allem auf Doldenblütlern anzutreffen. Im Palmengarten besucht er häufig Seseli-Blütenstände, aber auch wie viele andere Insekten Mannstreu-Arten (Eryngium giganteum u. a.). Der im Allgemeinen leicht zugängliche Pollen der Doldenblütler ist ein willkommenes Zubrot für den Bienenwolf. Meist lebt er jedoch nicht rein vegetarisch, sondern stellt anderen Blütenbesuchern nach, die er anschließend auffrisst. Die Weibchen legen über 200 Eier in die Nähe der Nester von (Wild-)Bienen wie beispielsweise Mauerbienen. Beim Hineinkrabbeln in ihr Nest befördern die Bienen die Käfereier unbeabsichtigt in das Nest hinein. Meist werden Nester von Solitärbienen, seltener von kleinen Bienenvölkern befallen. Die behaarten Larven des Käfers sind gegenüber Bienenstichen unempfindlich. Sie ernähren sich von den Larven und Puppen der Bienen, daher der Name Bienenwolf.

\section{Verwendete und weiterführende Literatur}

Bellmann, H. 2010: Steinbachs Naturführer Insekten. Stuttgart.

Harde, K. W. \& Severa, F. 1981: Der Kosmos Käferführer. - Stuttgart.

\section{Internetseiten}

http://www.wildbienen.de/wbi-r320.htm http://tierdoku.com/index.php?title=Immenk\%C3\%A4fer https://www.organische-chemie.ch/chemie/2010/feb/bienenwolf.shtm

https://offene-naturfuehrer.de/web/Bienenwolf_-_Philanthus_triangulum

https://nearbees.de/blog/auf-der-jagd-nach-bienen-der-bienenwolf/

\section{Anschrift der Autorin}

Dr. Hilke Steinecke, Palmengarten Frankfurt, Siesmayerstraße 61, 60323 Frankfurt, E-Mail: hilke.steinecke@stadt-frankfurt.de 